\title{
Spatial induction of genes encoding secreted proteins in micro-colonies of Aspergillus niger
}

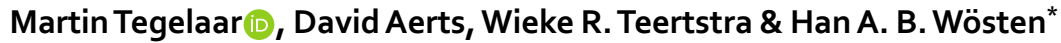

Aspergillus niger is used by the industry to produce enzymes and metabolites such as citric acid. In liquid cultures, it can grow as a dispersed mycelium or as micro-colonies with a width in the micrometer to millimeter range. Here, it was assessed whether expression of genes encoding secreted enzymes depends on mycelium morphology. To this end, expression of the reporter gene $g f p$ from the promoters of the glucoamylase gene glaA, the feruloyl esterase gene faeA and the $\alpha$-glucuronidase gene aguA was causally related to micro-colony size within a liquid shaken culture. Data could be fitted by hyperbolic functions, implying that the genes encoding these secreted proteins are expressed in a shell at the periphery of the micro-colony. The presence of such a shell was confirmed by confocal microscopy. Modelling predicted that the width of these zones was 13 to $156 \mu \mathrm{m}$ depending on growth medium and micro-colony diameter. Together, data indicate that the highest productive micro-colonies are those colonies that have a radius $\leq$ the width of the peripheral expression zone.

Filamentous fungi form mycelia that consist of a network of hyphae that grow at their tips and that branch sub-apically. Mycelia of aspergilli can reach a diameter in the sub-millimeter scale (micro-colonies) to centimeter scale (macro-colonies) depending on the size and the composition of the solid substrate ${ }^{1}$. For instance, Aspergillus forms micro-colonies on wheat kernels, whereas macro-colonies can be formed on fruits or bulbs of plants. In liquid shaken cultures, mycelium of Aspergillus grows dispersed, as micro-colonies, or in an intermediate state called clumps ${ }^{1}$. Dispersed mycelium consists of small networks of hyphae, while micro-colonies, also known as pellets, consist of a clear central and outer zone ${ }^{2}$. Notably, micro-colonies of Aspergillus niger produce more citric acid when compared to dispersed mycelium ${ }^{3,4}$. Why micro-colonies are more productive is not yet clear. It may be caused by the effect of the fungal morphology on the viscosity of the medium being high and low during dispersed growth and growth as micro-colonies, respectively ${ }^{5}$. At the same time, availability of oxygen and nutrients may impact productivity. Diffusion of these compounds would be sufficient in the case of dispersed mycelium while it would be limiting in the center of micro-colonies ${ }^{6}$.

So far, it is not known whether morphology of the mycelium affects production of secreted proteins. It was shown that micro-colonies within a liquid shaken culture are heterogeneous with respect to size and gene expres$\operatorname{sion}^{2}$. However, the relation between colony size and expression of genes encoding genes was not assessed. Therefore, we here studied whether micro-colony diameter affects expression of genes encoding secreted proteins in A. niger. To this end, the highly expressed genes encoding feruloyl esterase FaeA ${ }^{7,8}, \alpha$-glucuronidase AguA $A^{7,9}$ and glucoamylase $\mathrm{GlaA}^{7,10}$ were used as model genes of xylanolytic (FaeA, AguA) and amylolytic (GlaA) activity. Data show that these genes are expressed in the outer part of micro-colonies only. Results imply that small micro-colonies of A. niger produce more secreted proteins per biomass than large micro-colonies.

\section{Results}

Effect of mycelium transfer on micro-colony size. Wild-type strain N402 was grown in CMX (complete medium with xylose) for $16 \mathrm{~h}$ followed by a transfer to either CMX, CMM (complete medium with maltose), MMX (minimal medium with xylose) or MMM (minimal medium with maltose). Biomass of micro-colonies increased during a $4 \mathrm{~h}$ growth period from $0.6 \mathrm{~g}$ to $0.98,0.84,0.90$ and $0.76 \mathrm{~g}$, respectively. This was accompanied by an increase in mean micro-colony diameter from $948 \mu \mathrm{m}$ to 1091, 1065 and $987 \mu \mathrm{m}$, in the case of CMX, CMM and MMX respectively. In contrast, mean micro-colony diameter was reduced by $19 \mu \mathrm{m}$ after transfer to MMM. The size distribution of micro-colonies was also altered after transfer to the different media. In all cases, the mode was shifted to a higher diameter by $\sim 163 \mu \mathrm{m}$ (Fig. 1). However, a higher number of small micro-colonies were found as well upon transfer to MMX and MMM (Fig. 1D,E). This is illustrated by plotting the frequency of 


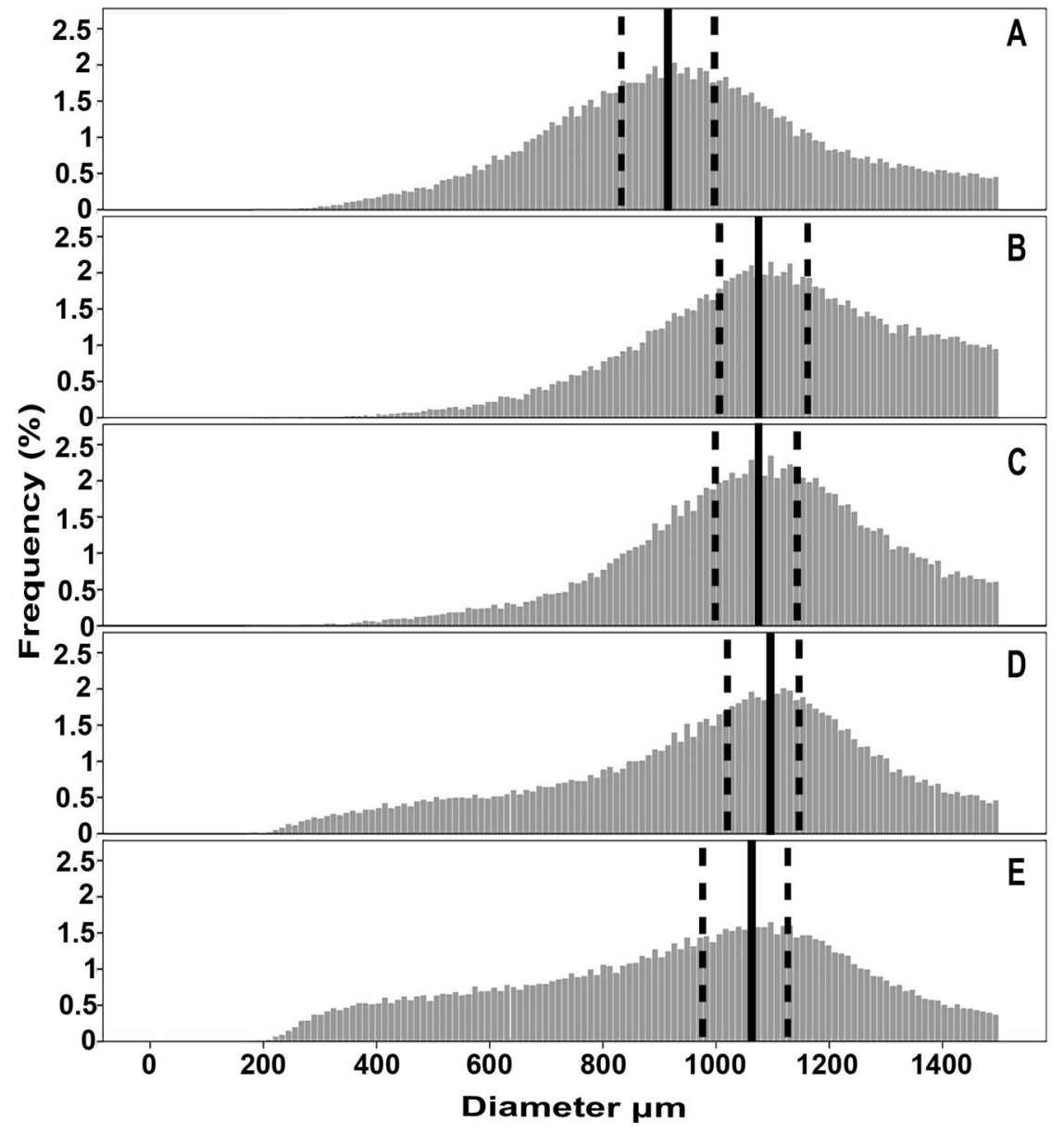

Figure 1. Size distribution of N402 micro-colonies that were not transferred to fresh medium (A) or were transferred from CMX to CMX (B), CMM (C), MMX (D) or MMM (E). Solid black lines flanked by dashed black lines represent the modes of the micro-colony populations and their $95 \%$ confidence intervals, respectively.

micro-colonies with diameters $<2.5^{\text {th }},>2.5^{\text {th }}<25^{\text {th }},>25^{\text {th }}<75^{\text {th }},>75^{\text {th }}<97.5^{\text {th }}$ and $>97.5^{\text {th }}$ percentile (Fig. 2). Transfer from CMX to MMX resulted in an increased proportion of micro-colonies within the smallest $2.5 \%$ of diameters as well as the largest $25 \%$ of diameters. At the same time, the frequency of micro-colonies of median sized micro-colonies was reduced. This shift was exacerbated when CMX grown micro-colonies were transferred to MMM, while transfer of micro-colonies from CMX to CMX only led to an increase in proportion of the micro-colonies within the largest $25 \%$ diameters and a decrease in proportion of micro-colonies within the median and smaller $25 \%$ of diameters. These findings were confirmed by multinomial logistic regression (data not shown). Together, data show that transfer to fresh medium promotes radial growth of N402 micro-colonies and, depending on the medium, can also result in partial micro-colony fragmentation.

Like wild-type N402, biomass of strains expressing $g f p$ from the glaA, faeA or $a g u A$ promoter increased upon transfer of $16 \mathrm{~h}$ old mycelium of non-inducing CM to inducing MM or CM. Biomass of the glaA::GFP, aguA::GFP and faeA::GFP strains had increased in MM from 0.71 to $0.78 \mathrm{~g}, 0.73$ to $0.83 \mathrm{~g}$ and 0.73 to $0.93 \mathrm{~g}$ during a $4 \mathrm{~h}$ period, respectively. When transferred to inducing CM, biomass of the glaA::GFP, aguA::GFP and faeA::GFP strains had increased to $0.85,1.47$ and $1.49 \mathrm{~g}$, respectively. Also in these strains, fragmentation occurred when mycelium was transferred to fresh medium (Fig. 3). In fact, mean micro-colony diameter of glaA::GFP (1361 $\mu \mathrm{m})$, aguA::GFP $(1142 \mu \mathrm{m})$ and faeA::GFP $(1230 \mu \mathrm{m})$ was reduced by 140,145 and $250 \mu \mathrm{m}$, respectively, after a $4 \mathrm{~h}$ transfer to inducing MM. This reduction was 180, 130 and $79 \mu \mathrm{m}$ for $g l a A:: G F P$, aguA::GFP and faeA::GFP, respectively, after transfer to $\mathrm{CM}$. Transfer of reporter strains to inducing $\mathrm{MM}$ or $\mathrm{CM}$ coincided with a decrease in the proportion of micro-colonies within the largest $25 \%$ of diameters and an increase in proportion of micro-colonies within the smallest $25 \%$ of diameters (Fig. 4). Proportions of median sized micro-colonies also decreased for the $a g u A:: G F P$ and faeA::GFP strains. In contrast, the glaA::GFP strain showed a marked increase in the proportion of median sized micro-colonies. Increase and decrease of proportions of size categories were of lesser magnitude after transfer of fluorescent strains to inducing CM. Diameters of small, median and large micro-colonies of the faeA::GFP and $a g u A:: G F P$ strains had reduced after transfer to inducing MM. For glaA::GFP, diameter of median and large microcolonies had also reduced after transfer to inducing MM. For the faeA::GFP and aguA::GFP strains transferred to inducing CM the smallest $2.5 \%$ of micro-colonies had increased in diameter, while small and median micro-colonies had reduced diameter. Large micro-colonies of aguA::GFP 


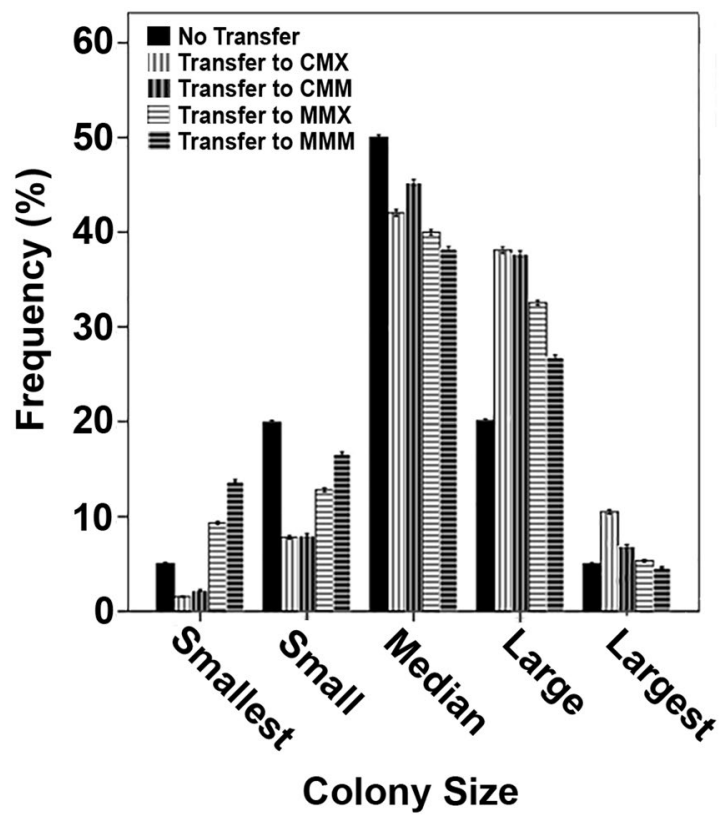

Figure 2. Proportions of size categories of N402 micro-colonies that were not transferred to fresh medium or were transferred from CMX to CMX, CMM, MMX or MMM. Error bars represent 95\% confidence intervals.

were also reduced in diameter, while large micro-colonies of faeA::GFP showed a slight increase in diameter. Micro-colony diameter of the glaA::GFP strain was reduced for median and large sized colonies but had increased for small micro-colonies. These findings were confirmed by multinomial logistic regression (data not shown). Together, biomass of the reporter strains increased after transfer to inducing medium but size of micro-colonies was reduced by micro-colony fragmentation.

Relation between micro-colony size and expression of genes encoding secreted proteins. The relation between micro-colony diameter and GFP fluorescence intensity was studied in the reporter strains glaA::GFP, aguA::GFP and faeA::GFP. As expected, non-induced strains or the wild-type strain N402 showed low or no fluorescence (Supplemental Fig. 1E). Total fluorescence intensity of reporter strains transferred to inducing medium increased quadratically with increasing diameter (Supplemental Fig. 2). Consequently, fluorescence intensity per micro-colony volume $\left(F V^{-1}\right)$ per diameter was best fitted by a hyperbolic function (Fig. 5A-E, Supplemental Fig. 3A-E). In this function, $F V^{-1}$ is maximal at the vertical asymptote $x_{b}$ of the hyperbolic function, while with increasing diameter $x, F V^{-1}$ decreases. Maximal $F V^{-1}$ of glaA::GFP, aguA::GFP and $f a e A:: G F P$ micro-colonies that had been induced in MM was found at a diameter of 288,211 and $26 \mu \mathrm{m}$, respectively (Table 1). When strains were induced in CM maximal $F V^{-1}$ was found at a diameter of 169 and $216 \mu \mathrm{m}$ for aguA::GFP and faeA::GFP, respectively. Conservative selection parameters derived from micro-colony profiles could lead to micro-colonies being omitted from analysis unintentionally. Therefore, the diameter where $F V^{-1}$ is maximal could be smaller in reality. This goes particularly for the CMM induced glaA::GFP strain as $a$ is equal to 0 , making $b$ an arbitrary number intersecting $\gamma$ with a minimum concurrent with the smallest recorded diameter. A hyperbolic function also has a horizontal asymptote $(\gamma)$ that approaches a steady state. For glaA::GFP, aguA::GFP and faeA::GFP induced in MM this steady state was reached at 1556, 1381 and $954 \mu \mathrm{m}$, respectively. The horizontal asymptote was reached at a diameter of 1254 and 1532 when aguA::GFP and faeA::GFP were induced in CM (Table 1). Slopes did not converge to a steady state in the case of CMM-induced glaA::GFP.

Increase in fluorescence intensity in the region between $x_{b}$ and $x_{\gamma}$ cannot be explained solely by increase in micro-colony volume when parameter $\alpha$ in Eq. 2 is shown not to be zero. This suggests that fluorescence is not evenly distributed over micro-colony volume but occurs in a concentric peripheral shell (Supplemental Fig. 4). Indeed, z-stacks of confocal laser scanning microscopy images showed a fluorescent concentric zone with a radius of 149,128 and $63 \mu \mathrm{m}$ for glaA::GFP, aguA::GFP and faeA::GFP when induced in MM and 69, 102 and $118 \mu \mathrm{m}$ when induced in CM, respectively (Table 2, Fig. 6; Supplemental Movies 1-3). To determine whether the width of the concentric shells depends on micro-colony size, fluorescence intensity per surface area was modeled (Fig. 5B-F; Supplemental Fig. 3B-F). In the case of MM, change in width was $<10 \%$. Modelling inferred that in the case of $\mathrm{MM}$ expression of $\mathrm{faeA}$ occurs in a relatively small concentric shell with a constant radius of $13 \mu \mathrm{m}$. In contrast, glaA is expressed in a concentric outer shell that increases in width from 144 to $157 \mu \mathrm{m}$ as micro-colonies increase in diameter from 288 to $1556 \mu \mathrm{m}$. On the other hand, $a g u A$ was predicted to be expressed in a concentric outer shell with a width decreasing from $105 \mu \mathrm{m}$ to $92 \mu \mathrm{m}$ in the 210 to $1381 \mu \mathrm{m}$ size range of micro-colonies. When reporter strains were induced in $\mathrm{CM}$, glaA was predicted to be expressed in a concentric outer shell that increases with a square root relationship. On the other hand, $a g u A$ was predicted to be expressed in a concentric outer shell that has a width decreasing from $85 \mu \mathrm{m}$ to $37 \mu \mathrm{m}$ in the 169 to $1245 \mu \mathrm{m}$ size range of micro-colonies. Moreover, fae $A$ was predicted to be expressed in a concentric outer shell with a width decreasing from $85 \mu \mathrm{m}$ to $31 \mu \mathrm{m}$ in 


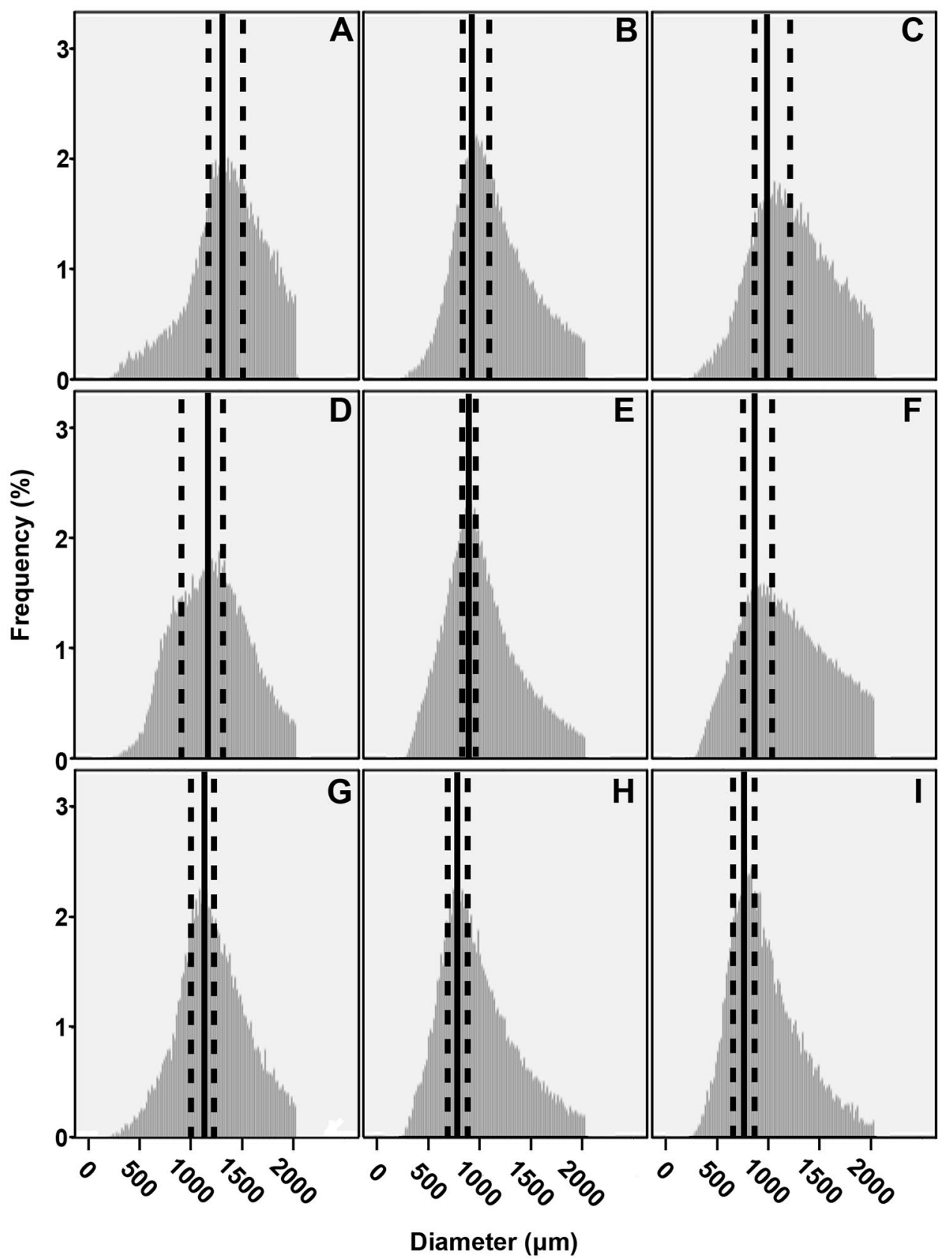

Figure 3. Size distribution of $g l a A:: G F P(\mathbf{A}, \mathbf{D}, \mathbf{G}), \operatorname{ag} u A:: G F P(\mathbf{B}, \mathbf{E}, \mathbf{H})$ and $f a e A:: G F P(\mathbf{C ,}, \mathbf{F}, \mathbf{I})$ micro-colonies that were or were not transferred from repressing CM $(\mathbf{A}-\mathbf{C})$ to inducing MM (D-F) or inducing CM $(\mathbf{G}-\mathbf{I})$. Solid black lines flanked by dashed black lines represent the modes and their $95 \%$ confidence intervals for the micro-colony populations, respectively.

the 230 to $1532 \mu \mathrm{m}$ size range of micro-colonies. Together, upon growth of the micro-colonies, the volume of the expression zone increases. At the same time its relative radius and volume decreases compared to the total diameter and volume of the pellet.

\section{Discussion}

Morphology of the mycelium in liquid cultures such as bioreactors depends on various factors including inoculum size, surface composition of the inoculum, medium composition, and mixing conditions ${ }^{1,7,11}$. In addition, presence of molecules such as chelating agents or anionic polymers may affect morphology. Here, it was shown for the first time that transfer of mycelium to fresh medium, in particular when changing the carbon source, also affects the morphology of the mycelium by inducing its fragmentation. Future studies will assess the underlying mechanisms and whether the observed fragmentation can be used to optimize productivity in liquid cultures.

So far, it had not been established how secretion of proteins relates to the morphology of the mycelium. Driouch et al. ${ }^{12}$ showed that $400-\mu \mathrm{m}$-wide micro-colonies express the glucoamylase gene glaA throughout the 


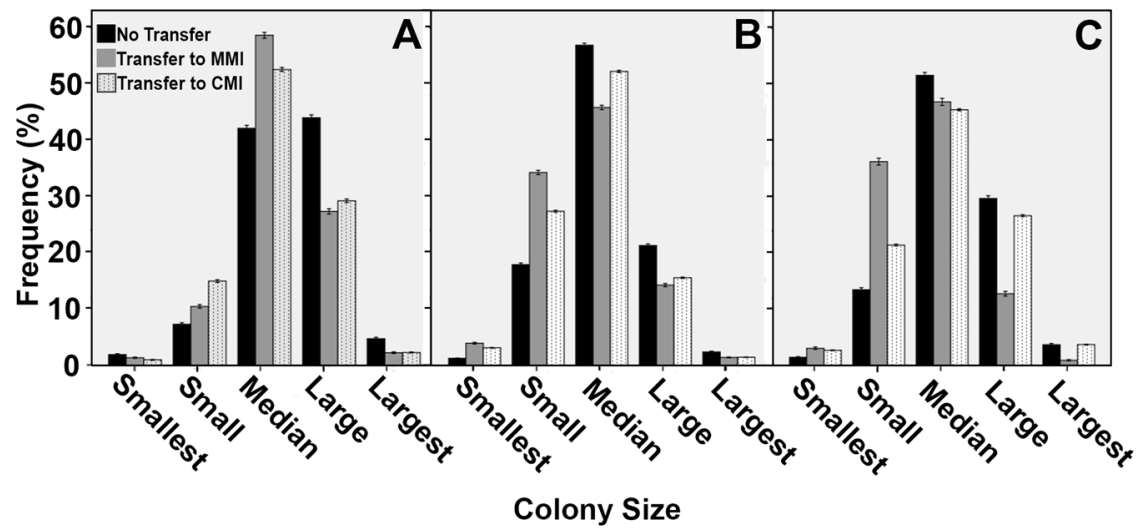

Figure 4. Proportions of size categories of $g l a A:: G F P(\mathbf{A})$, aguA::GFP (B) and $f a e A:: G F P(\mathbf{C})$ micro-colonies that were not or were transferred from repressing CM to inducing MM or CM. Error bars represent $95 \%$ confidence intervals.
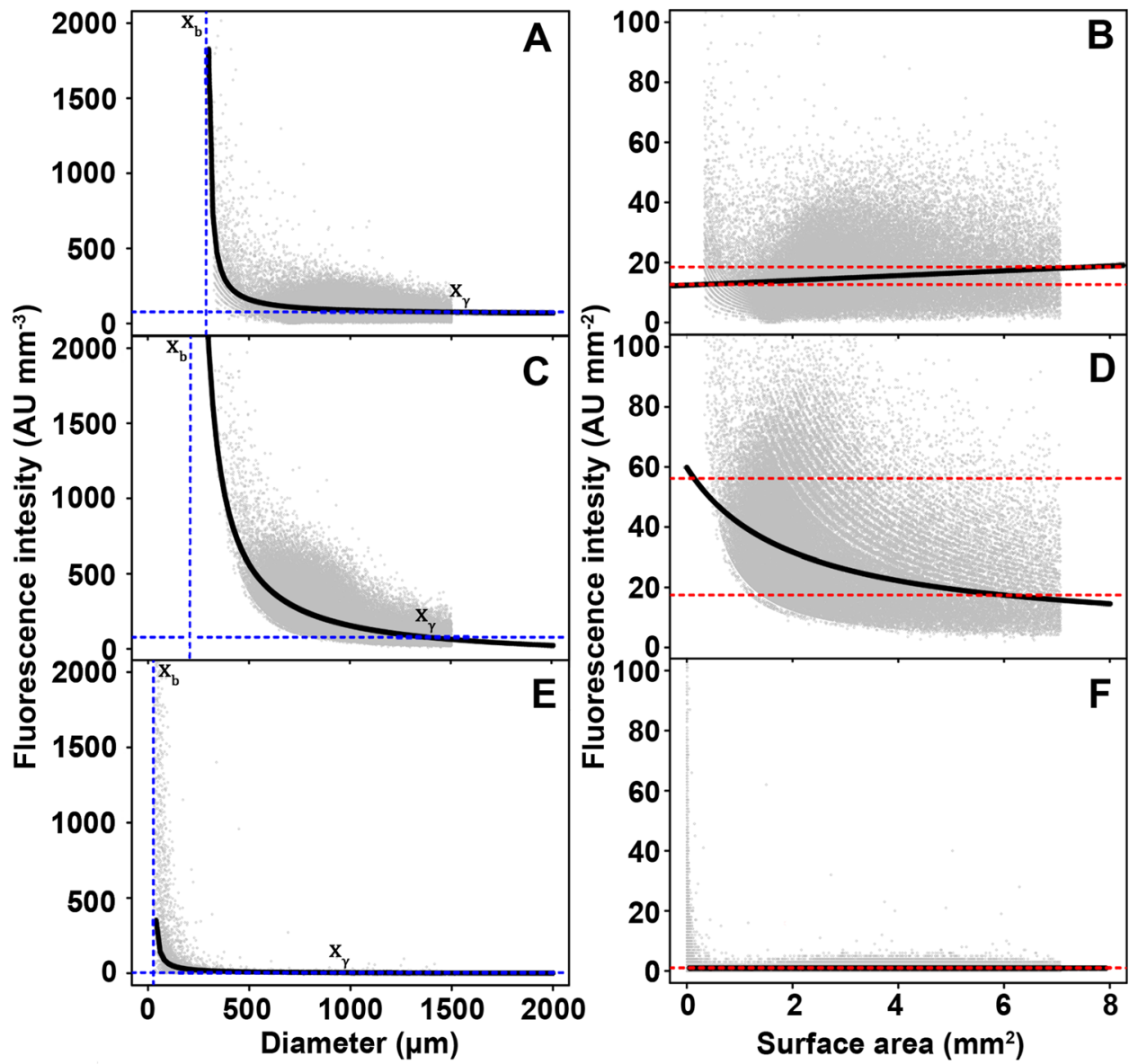

Figure 5. Relation between fluorescence per volume and pellet diameter $(\mathbf{A}, \mathbf{C}, \mathbf{E})$ and fluorescence per surface area and surface area $(\mathbf{B}, \mathbf{D}, \mathbf{F})$ of strains expressing $g f p$ from the $g l a A(\mathbf{A}, \mathbf{B}), a g u A(\mathbf{C}, \mathbf{D})$ and $f a e A(\mathbf{E}, \mathbf{F})$ promoter. Gray circles represent individual micro-colonies. The solid line represents the best fit as determined by quantile regression of the median. Horizonal blue dashed lines represent $F V^{-1}$ at steady state and vertical blue dashed lines represent the diameter where fluorescence intensity is maximal $\left(x_{b}\right)$. The intersect between $F V^{-1}$ at steady state and the lower $95 \%$ confidence limit of the best fit is denoted by $x_{\gamma}$. The red dashed lines (D-F) represent minimal and maximal fluorescence intensity $\mathrm{mm}^{-2}$ as predicted by EQ3. 


\begin{tabular}{|l|l|l|l|l|l|}
\hline Inducing medium & Promoter & $\mathbf{x}_{\mathbf{b}}(\boldsymbol{\mu m})$ & $\mathbf{x}_{\gamma}(\boldsymbol{\mu m})$ & $\mathbf{r}_{\gamma}(\boldsymbol{\mu m})$ & $\mathbf{r}_{\gamma} / \mathbf{0 . 5} \mathbf{x}_{\boldsymbol{\gamma}}(\%)$ \\
\hline \multirow{4}{*}{$M M$} & glaA & $288( \pm 8)$ & $1556(-80 /+109)$ & $156( \pm 0.8)$ & $21.5( \pm 0.1)$ \\
\cline { 2 - 6 } & aguA & $211( \pm 11)$ & $1381(-11 /+15)$ & $89( \pm 0.2)$ & $8.7( \pm 0.02)$ \\
\cline { 2 - 6 } & faeA & $26( \pm 0.3)$ & $954(-1.7 /+2.7)$ & $13( \pm 0.2)$ & $2.7( \pm 0.02)$ \\
\hline \multirow{3}{*}{$C M$} & glaA & - & - & - & - \\
\cline { 2 - 6 } & aguA & $169( \pm 3)$ & $1254( \pm 3)$ & $37( \pm 0.3)$ & $1( \pm 0.01)$ \\
\cline { 2 - 6 } & faeA & $230( \pm 12)$ & $1532( \pm 4)$ & $31( \pm 2)$ & $2( \pm 0.01)$ \\
\hline
\end{tabular}

Table 1. Values of relevant parameters from models describing the relation between micro-colony size and expression of $g f p$ from the $g l a A$, $a g u A$ and $f a e A$ promoters. Numbers between brackets indicate the $95 \%$ confidence interval. $\mathrm{x}_{\mathrm{b}}$ is micro-colony diameter where fluorescence intensity per volume $\left(\mathrm{FV}^{-1}\right)$ is maximal, $\mathrm{x}_{\mathrm{f}}$ represents the diameter where $\mathrm{FV}^{-1}$ remains constant when diameter increases, $r_{\gamma}$ is the predicted radius of the fluorescent band at this steady state and $\mathrm{r}_{\gamma} / 0.5 \mathrm{x}_{\gamma}$ represents the percentage of the radius of the micro-colony that shows fluorescence.

\begin{tabular}{|l|l|l|l|l|}
\hline Inducing medium & Promoter & Fluorescent radius $(\boldsymbol{\mu m})$ & Colony Diameter $(\boldsymbol{\mu m})$ & Fluorescent radius $(\%)$ \\
\hline \multirow{3}{*}{$M M$} & glaA & $149( \pm 19)$ & $1673( \pm 197)$ & $17,8(-4 /+5)$ \\
\cline { 2 - 6 } & aguA & $128( \pm 18)$ & $1332( \pm 256)$ & $19,2(-5 /+8)$ \\
\cline { 2 - 6 } & faeA & $63( \pm 17)$ & $1795( \pm 155)$ & $7(-2,4 /+2,8)$ \\
\hline \multirow{3}{*}{$C M$} & glaA & $69( \pm 7)$ & $1025( \pm 22)$ & $6,7( \pm 0,6)$ \\
\cline { 2 - 5 } & aguA & $102( \pm 16)$ & $1045( \pm 43)$ & $9,8( \pm 1,5)$ \\
\cline { 2 - 5 } & faeA & $118( \pm 13)$ & $1065( \pm 27)$ & $11,1( \pm 1,2)$ \\
\hline
\end{tabular}

Table 2. Radius of the expression zones of $g l a A$-, aguA-, and faeA-driven $g f p$ expression as shown by confocal microscopy. Numbers between brackets indicate the $95 \%$ confidence interval.

mycelium while expression in mm-sized microcolonies is only observed at the outer periphery. However, this study made use of the presence or absence of titanate micro-particles to control morphology. Therefore, changes in spatial gene expression may have been the result of the addition of the micro-particles. Here, we did not compare two cultures with different composition but rather made use of the heterogeneity of micro-colony morphology within a liquid shaken culture. Expression of $g l a A$, faeA and aguA per micro-colony volume generally decreased with increasing micro-colony size in a hyperbolic way, eventually approaching a constant expression per micro-colony volume. The hyperbolic relationship between colony diameter and fluorescence per volume is explained by expression taking place in a concentric shell at the periphery of the micro-colony. Expression in such a concentric shell was confirmed by fluorescence microscopy. When compared to confocal microscopy data models were reasonably accurate in predicting the fluorescent radius of $g l a A$ and $a g u A$ after transfer to inducing minimal medium; predicting the fluorescent radius of $g l a A:: G F P$ within its $95 \%$ confidence interval and underpredicting the fluorescent radius for $a g u A:: G F P$ by $16 \%$. Yet, the expression zone of faeA deviated by $72 \%$. For reporter strains transferred to inducing complete medium the fluorescent radius of the glaA::GFP strain could not be predicted because there were only $3 \%$ entirely fluorescent colonies in the culture. The fluorescent radius of aguA::GFP and faeA::GFP was under-predicted by $64 \%$ and $74 \%$, respectively. Underprediction of the expression zone of $f a e A$ may be caused by the relatively low expression of this gene, while underprediction of expression zones may also be caused by selection of micro-colonies in the confocal analysis that had not yet reached their steady state (i.e. they were too small). Particle analysis is therefore the preferred tool to assess expression zones because it takes into account all micro-colonies sizes in a culture instead of analysing single micro-colonies.

GFP expression was also found at the outer part of macro-colonies ${ }^{13}$. In contrast, the spatial expression in micro-colonies resulting from the $a g u A$ and fae $A$ promoters was not in accordance with those in macro-colonies ${ }^{13}$. These genes were found to be expressed throughout the macro-colony and the colony centre, respectively. The different spatial expression patterns in micro- and macro-colonies may be explained by inducer penetration. Micro-colonies in liquid shaken cultures are 3D structures. Penetration of the inducer into the centre of the colony may be difficult. In contrast, macro-colonies grown on solid medium are near $2 \mathrm{D}$ and therefore the inducer in the underlying medium has access to all zones of the colony. However, this does not explain why the radius of the fluorescent concentric shell of the faeA::GFP strain is relatively small when compared to the aguA::GFP strain despite the fact that both genes are induced by xylose. Possibly, these genes respond to different concentrations of the inducer.

The fact that expression of the genes encoding secreted proteins occurs at the periphery of the micro-colony in a shell with a relatively constant width means that the volume of this shell decreases relatively to the total volume of the micro-colony when the micro-colony becomes larger. Thus, cultures with uniform small micro-colonies (diameters at $x_{b}$ ) would be more productive than cultures with uniform large micro-colonies (diameters at) assuming similar biomass is formed per volume culture medium. Glucoamylase, $\alpha$-glucuronidase and feruloyl esterase production would then be 54, 56 and $91 \%$ less efficient at steady state diameters compared to micro-colonies with diameters at $x_{b}$ (i.e. diameter where expression per volume unit is maximal) if grown in minimal medium. In inducing complete medium, $\alpha$-glucuronidase and feruloyl esterase production would be 61 and $65 \%$ less efficient at steady state diameters compared to micro-colonies with diameters at $x_{b}$. 


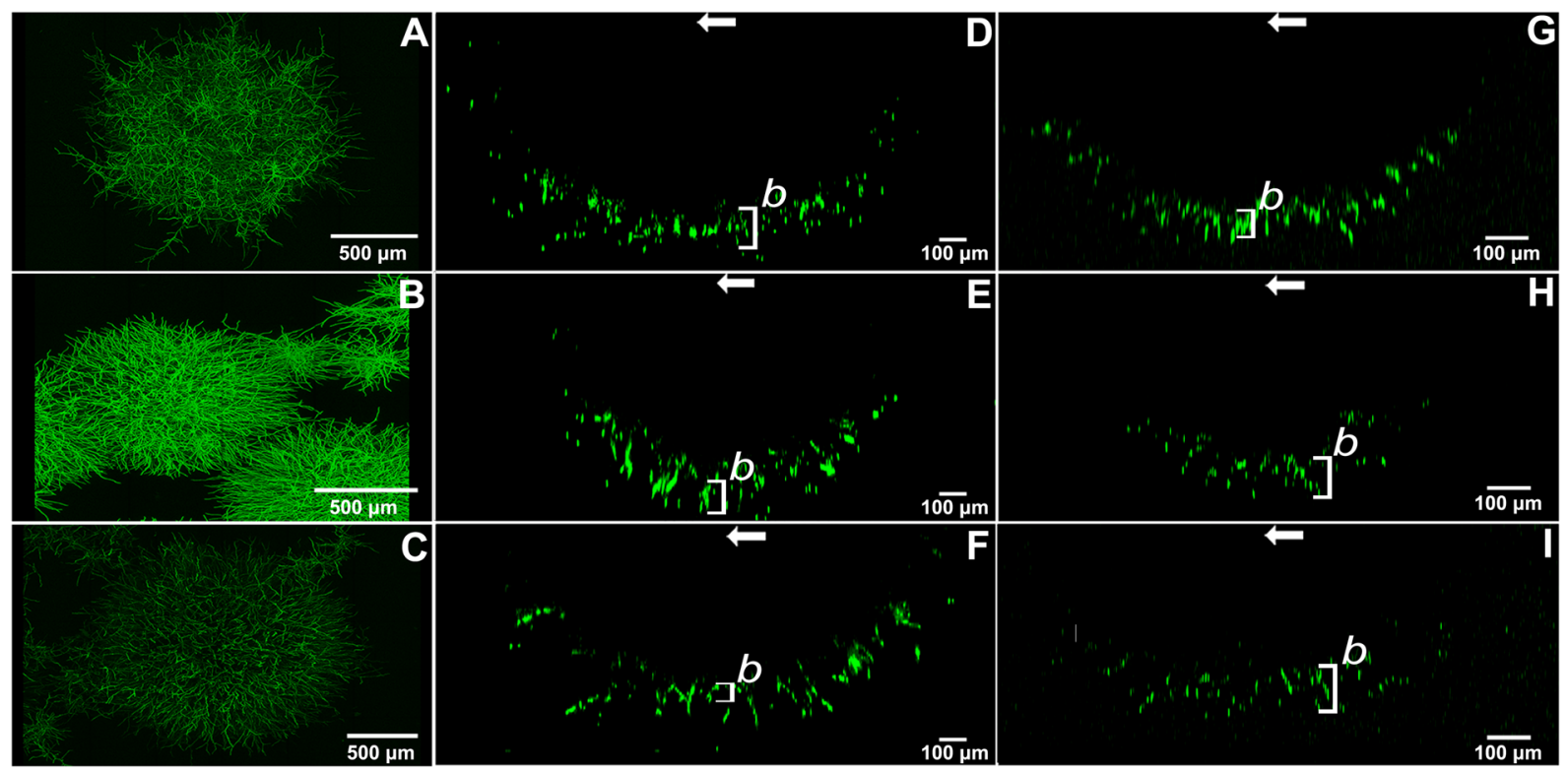

Figure 6. Maximum intensity $\mathrm{Z}$ projectionsremoved from the dataset because of confocal scanning images of the center of micro-colonies (A-C) and their respective YZ profiles from the center (D-I) of glaA::GFP(D-G), $a g u A:: G F P(\mathbf{E}-\mathbf{H})$ and $f a e A:: G F P(\mathbf{F}-\mathbf{I})$ that were induced in either MM (D,E,F) or CM (G-I). Arrows mark the center of the colony. Bars marked $b$ denote the mean thickness of the fluorescent shell.

\begin{tabular}{|l|l|l|l|}
\hline Strain & Parental Strain & Genotype & Reference \\
\hline N402 & CBS 120.49 & cspA1, amdS & 17 \\
\hline AR9\#2 & AB4.1 (derivative of N402) & $\begin{array}{l}\text { pyrG }{ }^{+}, \text {sGFP(S65T) under regulation of the } \\
\text { A. niger glaA promoter }\end{array}$ & 13 \\
\hline AV11\#3 & AB4.1 (derivative of N402) & $\begin{array}{l}\text { pyrG }{ }^{+}, \text {sGFP(S65T) under regulation of the } \\
\text { A. niger aguA promoter }\end{array}$ & 13 \\
\hline UU-A 005.4 & NW249 (derivative of N402) & $\begin{array}{l}\text { Nic-, leu- arg-, pyrG } \\
\text { regulation of the } \text { A. niger faeA } \text { promoter }\end{array}$ & 13 \\
\hline
\end{tabular}

Table 3. Strains used in this study.

\section{Methods}

Strains and culture conditions. Aspergillus niger strains N402, UU-A005.4, AR9\#2 and AV11\#3 were used in this study (Table 3). The former strain was used as a control, while the latter three strains (called faeA::GFP, $g l a A:: G F P$ and $a g u A:: G F P$ in this chapter) express $g f p$ from the $f a e A, g l a A$ and $a g u A$ promoter, respectively. Strains were grown at $30^{\circ} \mathrm{C}$ on minimal medium $\left(\mathrm{MM}^{14}\right.$,) with $25 \mathrm{mM}$ xylose and $1.5 \%$ agarose (MMXA). MMXA cultures were grown for three days, after which conidia were harvested using Saline-Tween $(0.8 \% \mathrm{NaCl}$ and $0.005 \%$ Tween-80). $250 \mathrm{ml}$ liquid cultures were inoculated with $1.25 * 10^{9}$ freshly harvested conidia and grown at $200 \mathrm{rpm}$ and $30^{\circ} \mathrm{C}$ in $1 \mathrm{~L}$ Erlenmeyer flasks in complete medium (CM) (MM containing $0.5 \%$ yeast extract and $0.2 \%$ enzymatically hydrolyzed casein) supplemented with either $25 \mathrm{mM}$ glucose (CMG; repressing aguA and faeA expression) or $25 \mathrm{mM}$ xylose (CMX; repressing glaA expression). Mycelium was harvested after $16 \mathrm{~h}$ and washed twice with PBS. Ten g of biomass (wet weight) was transferred to CMX or MM with $25 \mathrm{mM}$ xylose (MMX) (inducing $a g u A$ and $f a e A$ ) or to $\mathrm{CM}$ or MM with $25 \mathrm{mM}$ maltose (CMM, MMM) (inducing glaA expression). Biomass of cultures prior to transfer and after $4 \mathrm{~h}$ of growth in inducing medium was quantified after freeze drying.

Flow cytometry. Mycelium of liquid shaken cultures (biological triplicates) was fixed overnight at $4{ }^{\circ} \mathrm{C}$ in $4 \%$ paraformaldehyde in PBS, washed twice with PBS and taken up in $50 \mathrm{ml}$ PBS supplemented with $150 \mathrm{mM}$ glycine to quench autofluorescence. Diameter and fluorescence were analyzed using a BioSorter (Union Biometrica, Boston, MA, USA) equipped with a $2 \mathrm{~mm}$ nozzle (fluidics and optics core assembly (FOCA) 2000) and using a $488 \mathrm{~nm}$ solid state laser. At least 10000 particles per replica were analyzed for size and fluorescence measurements. Diameter of micro-colonies was determined from the time of flight (TOF) using $x=\frac{-b \pm \sqrt{b^{2}-4 a \cdot-T O F}}{2 a}(\mathrm{a}=0,00215762, \mathrm{~b}=5,433189436)$. This formula was inferred from the TOF of beads with diameters of $42 \mu \mathrm{m}, 250 \mu \mathrm{m}$ and $500 \mu \mathrm{m}$. Micro-colony volume (V) was calculated from the diameter assuming spherical morphology. To analyze the relation between micro-colony morphology and gene expression, particles with a TOF $<165$ and $>13005$ arbitrary units (AU) were removed from the dataset because they are outside the object size range of the FOCA (i.e. $<30 \mu \mathrm{m}$ and $>1500 \mu \mathrm{m}$ ). In addition, particles were removed with 
fluorescence peaks $<80$ AU. Peaks $>80$ AU but with a fluorescence width $<2000$ AU were also removed. Finally, particles were removed that had an integrated density $<25 \mathrm{AU}$ for the green, red and yellow channels. The integrated green value (in $\mathrm{AU}$ ) from the Biosorter was taken as a measure for fluorescence intensity (F). Micro-colonies of the non-fluorescent wild-type strain N402 were selected similarly but in this case particles were removed that had fluorescence peaks $<100$ AU. Additionally, integrated density of fluorescent signals was no longer a selection variable.

Differences in size distributions were quantified using two-sample Kolmogorov-Smirnov tests. Particles were distributed into five size categories based on diameter. Micro-colonies with diameters $<2.5^{\text {th }},>2.5^{\text {th }}<25^{\text {th }},>25^{\text {th }}<75^{\text {th }},>75^{\text {th }}<97.5^{\text {th }}$ and $>97.5^{\text {th }}$ percentile were designated smallest, small, median, large and largest, respectively. Differences in ratio between categories were analyzed using chi-square tests. Change of diameter after transfer to different media was described by resampling with 1000 -fold replacement. For each resample the mode was recorded as the central tendency. The median of the modes and the $2.5^{\text {th }}$ and $97.5^{\text {th }}$ percentile provided an approximation of the mode and its confidence intervals. For biomass quantification, micro-colonies were not fixed, filtered using coffee filters, transferred to $50 \mathrm{ml}$ falcon tubes and freeze-dried. Differences in dry weight were analyzed using Kruskal-Wallis $\mathrm{H}$ test.

Modelling GFP fluorescence relative to micro-colony diameter. Median fluorescence intensity data of micro-colonies that had been transferred to inducing medium were modelled with the R package quantreg ${ }^{15}$ using non-linear quantile regression. Data pertaining to the fluorescence intensity per unit of volume $\left(\mathrm{mm}^{3}\right)$ were best described by the non-linear hyperbolic functions Eqs. 1 and 2.

$$
\begin{gathered}
F V^{-1}=\frac{a}{x-b}+c \\
\frac{d F}{d V}=\frac{\alpha}{V}+\gamma
\end{gathered}
$$

In these equations, $F$ denotes fluorescence intensity and $V$ micro-colony volume $\left(\mathrm{mm}^{3}\right)$. In Eq. $1, x$ denotes micro-colony diameter $(\mu \mathrm{m})$, a represents the maximal fluorescence intensity per volume, $c$ denotes the arbitrary intercept of this function and $b$ represents the location of the vertical asymptote (i.e. the diameter $x_{b}$ of the micro-colony where fluorescence intensity per volume is maximal). In Eq. 2, $\alpha$ denotes the maximal fluorescence intensity per volume, while $\gamma$ represents a steady state between increase in fluorescence intensity and increase in volume. The x coordinate $x_{\gamma}$ of the intersect of the lower $95 \%$ confidence limit of Eq. 1 and $\gamma$ gives the diameter of micro-colonies that have reached this steady state.

Parameters $x_{b}$ and $x_{\gamma}$ were transformed to their corresponding surface areas, $S_{b}$ and $S_{\gamma}\left(\mathrm{mm}^{2}\right)$ and used as input in EQ3 to determine if fluorescence intensity increases proportionally to micro-colony size. Using $S_{b}$ and $S_{\gamma}$ as input for $S$, EQ3 gives the minimal $\left(F S_{b}^{-1}\right)$ and maximal $\left(F S_{\gamma}^{-1}\right)$ observed fluorescence intensity per unit of surface area. Equation 3.1 was used for glaA::GFP, while Eq. 3.2 was used for $a g u A:: G F P$ and faeA::GFP.

$$
\begin{gathered}
F S_{(b ; \gamma)}^{-1}=g S_{(b ; \gamma)}+i \\
F S_{(b ; \gamma)}^{-1}=\frac{g_{0}}{S_{(b ; \gamma)}-l}+i
\end{gathered}
$$

in which $S$ denotes surface area in $\mathrm{mm}^{2}$, while $i$ represents the intercept, $g_{0}$ the slope and $l$ the arbitrary location of the vertical asymptote. In this equation, any difference between $F S_{\gamma}^{-1}$ and $F S_{b}^{-1}$ in fluorescence per surface area is due to changing fluorescence intensity within the micro-colony, change of the radius of a hypothesized fluorescent shell, and/or the change in volume of this fluorescent shell due to increase in micro-colony size. Equation 4 was used to rule out that the difference between $\mathrm{FS}_{\gamma}^{-1}$ and $\mathrm{FS}_{b}^{-1}$ is explained by the change in volume of the fluorescent shell. In this equation the change of fluorescence intensity per surface area is given by the derivative of $F S^{-1}: f^{\prime}(S)$.

$$
\Delta F S_{b \gamma}^{-1}=\int_{S_{\mathrm{b}}}^{S_{\gamma}} f^{\prime}(S)-\int_{V_{S_{b}}}^{V_{S_{\gamma}}} f^{\prime}\left(S_{v}\right)
$$

where $V_{s_{b}}$ and $V_{s_{\gamma}}$ represent the minimal and maximal volume of the fluorescent shell. For derivative $f^{\prime}\left(S_{v}\right)$ the variable $S$ has been substituted by $\frac{S^{\frac{3}{2}}-\left(S-S_{b}\right)^{\frac{3}{2}}}{6 \sqrt{2^{T}}}$ to describe the volume of the fluorescent shell. When $\Delta F S_{b \gamma}^{-1}$ does not equal 0 , fluorescence intensity $\mathrm{mm}^{6 \sqrt{2}}$ can only be explained by change in width of the fluorescent shell in the $\mathrm{z}$-axis or its fluorescence intensity. The surface and volume independent change of fluorescence intensity of the fluorescent shell is revealed by Eq. 5.1 and Eq. 5.2, which were used for glaA::GFP and aguA::GFP and faeA::GFP, respectively.

$$
F_{i}=\int_{S_{b}}^{S_{\gamma}} \frac{\Delta F S_{b \gamma}^{-1}}{S_{\gamma}-S_{b}} S-\frac{\Delta F S_{b \gamma}^{-1}}{S_{\gamma}-S_{b}} S_{b}
$$




$$
F_{i}=\int_{S_{b}}^{S_{\gamma}} \frac{\frac{\left(F S^{-1}-F S_{b}^{-1}\right)}{\left(S-S_{b}\right)} \frac{\left(S-S_{\gamma}\right)}{\left(F S^{-1}-F S_{\gamma}^{-1}\right)}}{\frac{\left(\sqrt{g}+i+\Delta F S_{b \gamma}^{-1}-F S_{\gamma}^{-1}\right)}{\left(\sqrt{g}+l-S_{b}\right)} \frac{\left(\sqrt{g}+l-S_{\gamma}\right)}{\left(\sqrt{g}+i-F S_{b}^{-1}\right)}}-F S_{b}^{-1}
$$

When $F_{i}$ is substituted in Eq. 6, the hypothesized increase of fluorescent radius $\mathrm{z}$ is found.

$$
\begin{aligned}
V_{s} & =\frac{F_{i}+\int_{S_{b}}^{S_{\gamma}} F S_{(b: \gamma)}^{-1}}{m} \\
V_{s_{b}} & =\frac{\int_{S_{b}}^{S_{\gamma}} F S_{(b: \gamma)}^{-1}}{m} \\
V_{s} \cdot 10^{9} & =\frac{1}{6} \pi\left(x^{3}-\left(x-x_{b}\right)^{3}\right) \\
V_{s_{b}} \cdot 10^{9} & =\frac{1}{6} \pi\left(x^{3}-\left(x-x_{b}\right)^{3}\right) \\
\mathrm{z} & =\frac{x_{b} \cdot x_{V_{s}}}{2 x_{V_{s_{b}}}}-0.5 x_{V_{s_{b}}}
\end{aligned}
$$

where $m$ is the relation between fluorescence intensity and volume of the fluorescent shell. $F S_{(\gamma)}^{-1}$ or $F S_{(b)}^{-1}$ should be input in Eq. 6 for fluorescent shells that decrease or increase in width with surface area, respectively. The relative fluorescent radius of a micro-colony is then given by Eq. 7 .

$$
I_{x}=\frac{0.5 x_{V_{s_{b}}}+z}{0.5 x}
$$

Finally, the percentage of fluorescent micro-colony volume at the steady state is given by Eq. 8 , in which $\mathrm{V}_{0}$ is the total volume of the micro-colony and $V_{1}$ is the non-fluorescent volume.

$$
\begin{aligned}
V_{0} & =\frac{1}{6} \pi(x)^{3} \\
V_{1} & =\frac{1}{6} \pi\left(\left(x-I_{x} \cdot x\right)^{3}\right) \\
I_{v} & =\frac{V_{0}-V_{1}}{V_{0}}
\end{aligned}
$$

When micro-colonies were transferred to $\mathrm{CM}$, equations described above remained valid for the aguA::GFP and faeA::GFP strains. However, the glaA::GFP strain was now best described by a horizontal line at $\gamma$, with $b$ as an arbitrary intersect of this line.

Confocal laser scanning microscopy. Fluorescence of GFP was localized in micro-colonies using a DMI 6000 CS AFC confocal microscope (Leica, Mannheim, Germany). Micro-colonies were fixed and washed (see above), transferred into a glass bottom dish (Cellview ${ }^{\mathrm{TM}}$, Greiner Bio-One, Frickenhausen, Germany, PS, 35/10 $\mathrm{MM}$ ) and embedded in $1 \%$ low melting point agarose at $45^{\circ} \mathrm{C}$. Micro-colonies were imaged at 20x magnification (HC PL FLUOTAR L $20 \times / 0,40 \mathrm{DRY})$. GFP was excited by white light laser at $472 \mathrm{~nm}$ using $50 \%$ laser intensity $\left(0.1 \mathrm{~kW} / \mathrm{cm}^{2}\right)$ and a pixel dwell time of $72 \mathrm{~ns}$. Fluorescent light emission was detected with hybrid detectors in the range of 490-525 nm. Pinhole size was 1 Airy unit. In all cases, z-stacks were made of imaged micro-colonies using 100 slices with a slice thickness of $5.4-10 \mu \mathrm{m}$. Image analysis was performed with Fiji ${ }^{16}$.

\section{Data availability}

Data are available for readers upon request.

Received: 31 May 2019; Accepted: 13 January 2020;

Published online: 30 January 2020

\section{References}

1. Krijgsheld, P. et al. Development in Aspergillus. Stud. Mycol. 74, 1-29 (2013).

2. de Bekker, C., van Veluw, G. J., Vinck, A., Wiebenga, L. A. \& Wösten, H. A. B. Heterogeneity of Aspergillus niger microcolonies in liquid shaken cultures. Appl. Environ. Microbiol. 77, 1263-1267 (2011).

3. Clark, D. S. Submerged citric acid fermentation of ferrocyanide-treated beet molasses: morphology of pellets of Aspergillus niger. Can. J. Microbiol. 8, 133-136 (1962).

4. Clark, D. S., Ito, K. \& Horitsu, H. Effect of manganese and other heavy metals on submerged citric acid fermentation of molasses. Biotechnol. Bioeng. 8, 465-471 (1966).

5. Bhargava, S., Wenger, K. S. \& Marten, M. R. Pulsed addition of limiting-carbon during Aspergillus oryzae fermentation leads to improved productivity of a recombinant enzyme. Biotechnol. Bioeng. 82, 111-117 (2003).

6. Hille, A., Neu, T. R., Hempel, D. C. \& Horn, H. Effective diffusivities and mass fluxes in fungal biopellets. Biotechnol. Bioeng. 103, $1202-1213(2009)$. 
7. Lu, X. et al. The intra- and extracellular proteome of Aspergillus niger growing on defined medium with xylose or maltose as carbon substrate. Microb. Cell Fact. 9, 23 (2010).

8. de Vries, R. P. et al. The faeA genes from Aspergillus niger and Aspergillus tubigensis encode ferulic acid esterases involved in the degradation of complex cell wall polysaccharides. Appl. Environ. Microbiol. 63, 4638-4644 (1997).

9. de Vries, R. P., Poulsen, C. H., Madrid, S. \& Visser, J. aguA, the gene encoding an extracellular $\alpha$-glucuronidase from Aspergillus tubingensis, is specifically induced on xylose and not on glucuronic acid. J. Bacteriol. 180, 243-249 (1998).

10. Fowler, T., Berka, R. M. \& Ward, M. Regulation of the glaA gene of Aspergillus niger. Curr. Genet. 18, 537-545 (1990).

11. Wösten, H. A. B., van Veluw, G. J., de Bekker, C. \& Krijgsheld, P. Heterogeneity in the mycelium: implications for the use of fungi as cell factories. Biotechnol. Lett. 35, 1155-1164 (2013).

12. Driouch, H., Hänsch, R., Wucherpfennig, T., Krull, R. \& Wittmann, C. Improved enzyme production by bio-pellets of Aspergillus niger: targeted morphology engineering using titanate microparticles. Biotechnol. Bioeng. 109, 462-471 (2012).

13. Vinck, A. et al. Heterogenic expression of genes encoding secreted proteins at the periphery of Aspergillus niger colonies. Environ. Microbiol. 13, 216-225 (2011).

14. de Vries, R. P. et al. A new black Aspergillus species, A. vadensis, is a promising host for homologous and heterologous protein production. Appl. Environ. Microbiol. 70, 3954-3959 (2004).

15. Koenker, R. Quantreg: Quantile Regression. R package version 5.33. https://CRAN.Rproject.org/package=quantreg (2017).

16. Schindelin, J. et al. Fiji: an open-source platform for biological-image analysis. Nat. Meth. 9, 676-682 (2012).

17. Bos, C. J. et al. Genetic analysis and the construction of master strains for assignment of genes to six linkage groups in Aspergillus niger. Curr. Genet. 14, 437-43 (1988).

\section{Acknowledgements}

This study was financed by the Netherlands Organization for Scientific Research (NWO) and the Topsector Energie.

\section{Author contributions}

M.T., D.A. and W.T. performed, analyzed, interpreted, and designed the experiments; H.W. supervised the project; M.T., D.A. and H.W. wrote the manuscript.

\section{Competing interests}

The authors declare no competing interests.

\section{Additional information}

Supplementary information is available for this paper at https://doi.org/10.1038/s41598-020-58535-0.

Correspondence and requests for materials should be addressed to H.A.B.W.

Reprints and permissions information is available at www.nature.com/reprints.

Publisher's note Springer Nature remains neutral with regard to jurisdictional claims in published maps and institutional affiliations.

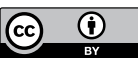

Open Access This article is licensed under a Creative Commons Attribution 4.0 International

License, which permits use, sharing, adaptation, distribution and reproduction in any medium or format, as long as you give appropriate credit to the original author(s) and the source, provide a link to the Creative Commons license, and indicate if changes were made. The images or other third party material in this article are included in the article's Creative Commons license, unless indicated otherwise in a credit line to the material. If material is not included in the article's Creative Commons license and your intended use is not permitted by statutory regulation or exceeds the permitted use, you will need to obtain permission directly from the copyright holder. To view a copy of this license, visit http://creativecommons.org/licenses/by/4.0/.

(C) The Author(s) 2020 